\title{
Rerouting an Organocatalytic Reaction by Intercepting its Reactive Intermediates
}

\author{
Santosh C. Gadekar ${ }^{\ddagger}$, Vasudevan Dhayalan ${ }^{\ddagger}$, Ashim Nandi, Inbal L. Zak, Shahar Barkai, Meital \\ Shema Mizrachi, Sebastian Kozuch and Anat Milo*
}

Department of Chemistry, Ben-Gurion University of the Negev, Beer Sheva, Israel.

\section{Placeholder}

\begin{abstract}
Reactive intermediates are key to halting and promoting chemical transformations, however due to their elusive nature, they are seldom harnessed for reaction design. Herein, we describe studies aimed at stabilizing reactive intermediates in the $\mathrm{N}$-heterocyclic carbene (NHC) catalytic cycle, which enabled fully shutting down the known benzoin coupling pathway, while rerouting its intermediates toward deuteration. The reversible nature of NHC catalysis and the selective stabilization of reaction intermediates facilitated clean hydrogen-deuterium exchange reactions of aromatic aldehydes by $\mathrm{D}_{2} \mathrm{O}$, even for challenging electron withdrawing substrates. The addition of catalytic amounts of phenyl boronic acid was used to further stabilize highly reactive intermediates and mitigate the formation of benzoin coupling byproducts. The mechanistic understanding at the foundation of this work resulted in unprecedented mild conditions with base and catalyst loadings as low as $0.1 \mathrm{~mol} \%$, and a scalable deuteration reaction applicable to a broad substrate scope with outstanding functional group tolerance. More importantly, adopting this approach enabled the construction of a machine-learning derived guideline for identifying the appropriate catalyst and conditions for different substrates based on a logistic regression classification model. Experimental studies combined with machine learning and computational methods shed light on the non-trivial mechanistic underpinnings of this reaction.
\end{abstract}

Organocatalytic reactions are often plagued by low energy barriers between competing pathways. Consequently, despite their vast potential to provide a greener alternative to metal based catalysis, they often suffer from reproducibility issues and limited substrate scopes. Identifying the structural origin of organocatalytic reactivity and selectivity can provide a knowledge-driven approach to reaction design and optimization, unlocking the full potential of organocatalysis. ${ }^{1-7} \mathrm{By}$ intercepting reactive intermediates and controlling their stability, one can not only improve the efficiency of an organocatalytic transformation, but also take advantage of those seemingly undesirable competing pathways. To this end, we sought to gain control over the intermediates involved in $\mathrm{N}$-heterocyclic carbene (NHC) organocatalysis and reroute them to deuterium incorporation by hydrogen- deuterium exchange using $\mathrm{D}_{2} \mathrm{O}$. We hypothesized that intercepting a stabilized aldehyde intermediate could serve as a facile entry point for introducing a deuterium label.

The value of selective deuterium labeling of aromatic aldehydes cannot be overstated. These ubiquitous building blocks are perfect candidates for introducing deuterium labels into biologically active compounds through synthesis or late-stage functionalization. The utility of deuterium labeled compounds has been widely demonstrated in absorption, distribution, metabolism, and excretion (ADME) studies, and has been implicated in boosting metabolic and pharmacokinetic efficacy. ${ }^{8-11}$ Furthermore, deuterated compounds are of great value for nuclear magnetic resonance spectroscopy, ${ }^{12}$ mass spectrometry ${ }^{13-14}$ and the investigation of reaction mechanisms. ${ }^{10}$ Despite recent advances, ${ }^{15-23}$ current approaches for the preparation of deuterium labeled aldehydes (aldehyde- $d$ ) are still limited by unselective reactions, harsh reaction conditions, restricted substrate scopes and poor deuterium incorporation. These limitations particularly affect highly substituted and electrophilic aldehydes as they can readily undergo side reactions. Yet, it is particularly useful to identify conditions for polar and functionalized compounds because there is a growing recognition in the field of drug design that while these compounds are more likely to fail to undergo catalytic reactions, they are also more likely to become successful drug candidates. ${ }^{24}$ These challenging substrates provided an opportunity to appropriate the NHCcatalyzed benzoin reaction and direct its activated aldehyde intermediates towards deuteration.

Aldehyde deuteration has been gaining attention, ${ }^{15-20}$ and several recent works applied NHCs as organocatalysts in these reactions. ${ }^{21-23}$ Bouffard, Bertrand, Yan and co-workers used mesoionic carbenes with methanol- $d$ as the deuterating reagent, ${ }^{15}$ while Chen, Zhang, Wang and coworkers as well as Sawama, Sajiki and coworkers used imidazolium and triazolium NHCs with $\mathrm{D}_{2} \mathrm{O}$ as the deuterating reagent. ${ }^{22-23}$ In our previous work, we used boronic acids to stabilize acyl anion adducts in the NHC catalyzed benzoin reaction. ${ }^{25}$ Herein, we exploit mechanistic insights on the stabilization of intermediates in NHC catalysis for the selective deuteration of aromatic aldehydes. Because we approached the problem from an entirely mechanistic perspective, this work ultimately served to elucidate the underpinnings of the 
aforementioned methodologies. Moreover, this approach led us to identify much milder conditions with catalyst and base loading as low as $0.1 \mathrm{~mol} \%$, achieving higher reaction yields and cleaner reactions. The use of phenyl boronic acid to stabilize more reactive acyl anion adducts enabled the selective deuteration of challenging substrates, particularly those containing electron withdrawing groups. Notably, the mechanistic foundation of this work resulted in a machine-learning derived guideline for selecting suitable reaction conditions for different substrates, abating extensive trail-and-error optimizations for each new substrate.
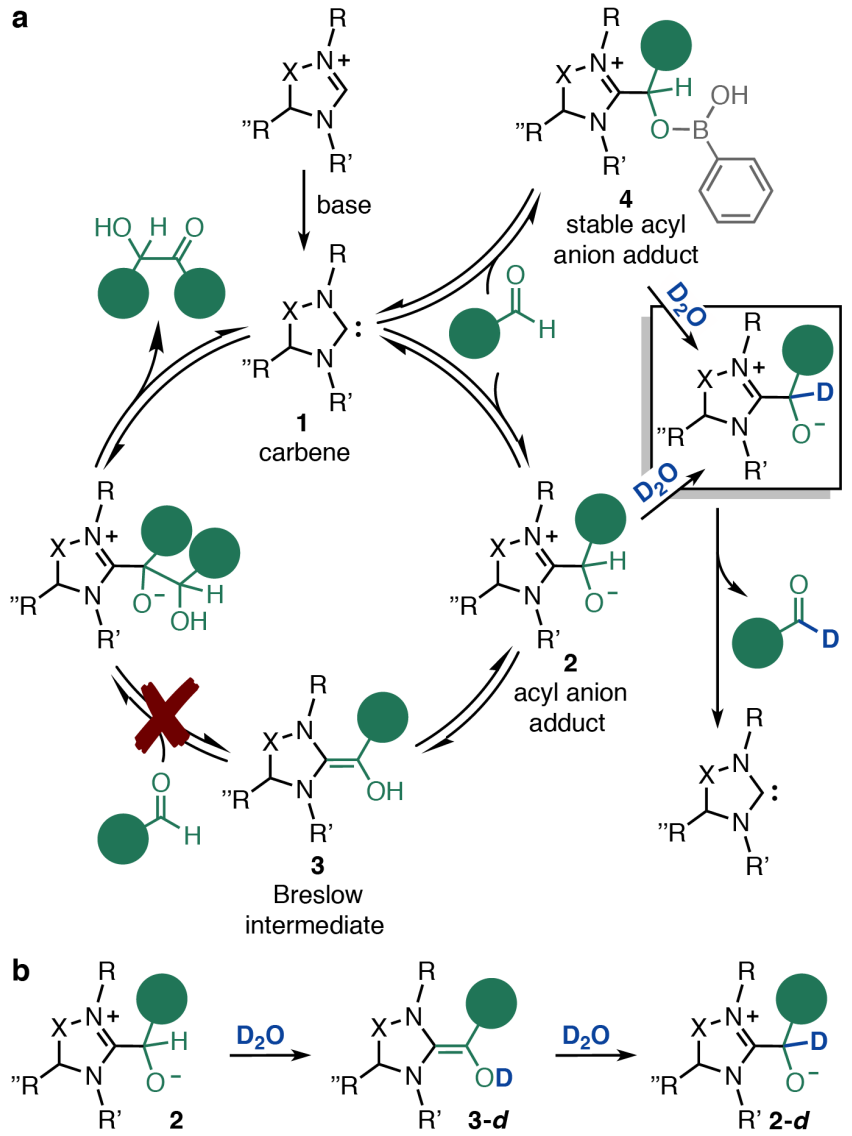

Figure 1. a. Putative catalytic cycle for the benzoin condensation reaction. The goal of this work was the suppression of the benzoin reaction and interception of its intermediates for the deuteration of aldehydes. b. Proposed process for the deuteration of aldehydes using NHC catalysts.

In the commonly accepted mechanism for the NHC catalyzed benzoin condensation, an azolium salt undergoes deprotonation with base to form carbene $\mathbf{1}$ (figure 1.a.). ${ }^{26-28} \mathrm{~A}$ nucleophilic attack of species $\mathbf{1}$ on an aldehyde generates the acyl anion adduct 2. Subsequent protonation/deprotonation lead to the formation of an amino enol 3 known as the Breslow intermediate, which entails the inversion of polarity (umpolung) at carbon. This intermediate can then attack another aldehyde to form the benzoin product. The steps involved in the NHC catalyzed benzoin reaction are reversible, ${ }^{25,29-31}$ thus lend themselves to the interception of reactive intermediates. More than 50 year after Breslow proposed an umpolung intermediate, ${ }^{32}$ Berkessel and coworkers succeeded in the elusive task of stabilizing and characterizing it using saturated imidazolium activated NHCs. ${ }^{33}$ O'Donoghue, Smith and coworkers studied the effect of different pyrroli- dine based triazolium NHC organocatalysts on the stability of 3-(hydroxy-benzyl)azolium salts, which are protonated acyl anion adducts. ${ }^{34-35}$ Based on these literature precedents, we assumed that saturated imidazolium or pyrrolidine based triazolium NHC salts would be ideal candidates for intercepting the acyl anion adduct 2 . The stabilization of this adduct and its exclusive reaction with $\mathrm{D}_{2} \mathrm{O}$ would presumably afford the in situ generation of a deuterated Breslow intermediate, which would in turn attack $\mathrm{D}_{2} \mathrm{O}$ and decompose to selectively provide aldehyde- $d$ (figure 1.b.).

Unravelling multifaceted reactivity and stabilizing intermediates. In this work, we disclose an approach to control the selective formation of aromatic aldehyde- $d$ by suppressing the formation of benzoin homo-coupling products. The main challenge was controlling the formation of the benzoin product, while still boosting the reactivity of the Breslow intermediate toward $\mathrm{D}_{2} \mathrm{O}$. Achieving this goal was multifaceted because the formation of the undesired homocoupling product and the deuteration reaction were both substrate dependent (figure 2). On the one hand, the formation of 2-substituted benzaldehyde homo-coupling products is known to be sluggish, likely due to the stability of the acyl anion adduct (2 figure 1). ${ }^{35}$ Therefore, we expected that these substrates would not suffer from homo-coupling byproducts, an assumption that was confirmed by preliminary experiments with a 2,6-diisopropylphenyl substituted imidazolium catalyst (SIPr); however, it was revealed that they only underwent partial deuteration. On the other hand, 3and 4-substituted benzaldehydes easily underwent deuteration; yet, their homo-coupling benzoin reaction was also facile, especially for aldehydes with electron withdrawing substituents. Thus, we studied these substrate classes separately to uncover the underpinnings of the reactivity observed for each with the aim of providing tailored solutions.

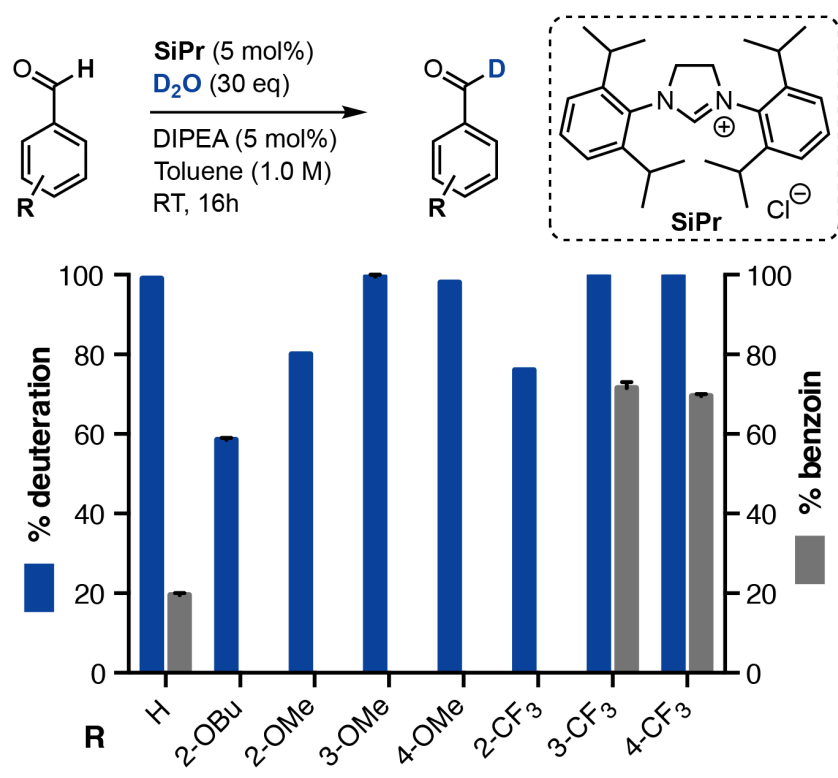

Figure 2. Initial reactions with SIPr revealed the substrate dependence of competing deuteration and benzoin reactions.

To understand whether the extent of deuterium incorporation for 2-substituted benzaldehydes was contingent on electronic or steric effects, we sought to correlate deuterium incorporation with benzaldehyde structural features. 


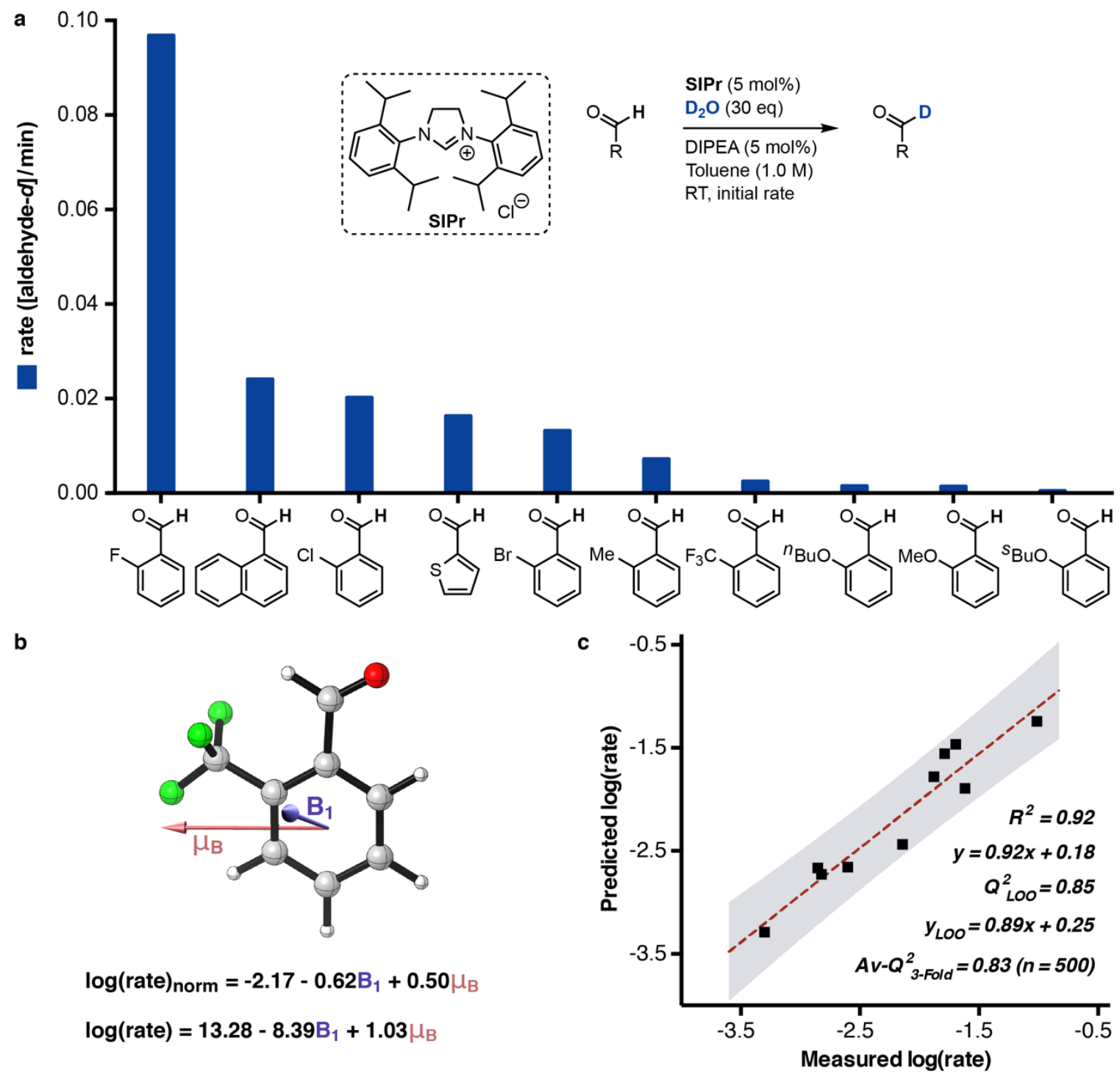

Figure 3. a. Initial deuteration rate of different 2-substituted aldehydes $(\mathrm{M} / \mathrm{min})$. b. Equation for the $\log ($ rate $)$ of deuteration of 2-substituted aldehydes including two molecular descriptors: $\mathrm{B}_{1}$, the minimal width of the aldehyde, and $\mu_{\mathrm{B}}$, the component of the dipole moment in the direction of the maximal width on the ring plane (normalized parameters were obtained by subtracting the mean and dividing by the standard deviation). c. Measured versus predicted $\log \left(\right.$ rate) of deuteration. Goodness-of-fit represented by $\mathrm{R}^{2}$, $\mathrm{Q}^{2}$ for a leave-one-out validation, and the average $\mathrm{Q}^{2}$ of a 3-fold validation performed 500 times on randomized sets with $90 \%$ prediction interval in grey.

Therefore, we turned to explore the initial rates of reaction for several 2-substituted benzaldehydes (figure 3.a.). The trend was not clearly steric or electronic, thus, multivariate regression was applied to identify substrate features that influence deuteration rates. We found that the Sterimol ${ }^{36}$ minimal width of the substrate $\left(\mathrm{B}_{1}\right)$ combined with the width component of the dipole moment $(\mu \mathrm{B})$ could predict the deuteration rate for a diverse set of 2 -substituted substrates (Figure 3.b-c.). Slower rates are observed for wider substrates (with larger $\mathrm{B}_{1}$ values) with greater dipole moments (more negative $\mu_{B}$ values), which implies that the rate of deuteration is dependent on the balance between the size and electronegativity of substituents at the 2-position. This result also indicates that in order to identify a more efficient catalyst for the substrates that were not fully deuterated, a simple modification of either the steric or electronic character of the catalyst structure would not suffice.
Based on the assumption that pyrrolidine based triazolium NHC catalysts (TACs) form more reactive acyl anion adducts compared to imidazolium NHCs, ${ }^{34-35,37-43}$ we prepared a family of TACs containing different aryl rings, for which both steric and electronic properties could be readily modified. The activity of these catalysts was evaluated with 2- ${ }^{n}$ butoxybenzaldehyde because it was a substrate that led to low deuterium incorporation using SIPr as catalyst (figure 4.a.). Similarly to the trend for different substrates, the influence of the catalyst aryl ring on the level of deuteration was not purely steric or electronic. Furthermore, using parameters derived exclusively from the precatalyst ground state structure led to overfitted or ineffective models. To overcome this limitation, we turned to the proposed reaction mechanism, which would entail the formation of a Breslow intermediate prior to the deuteration step. 

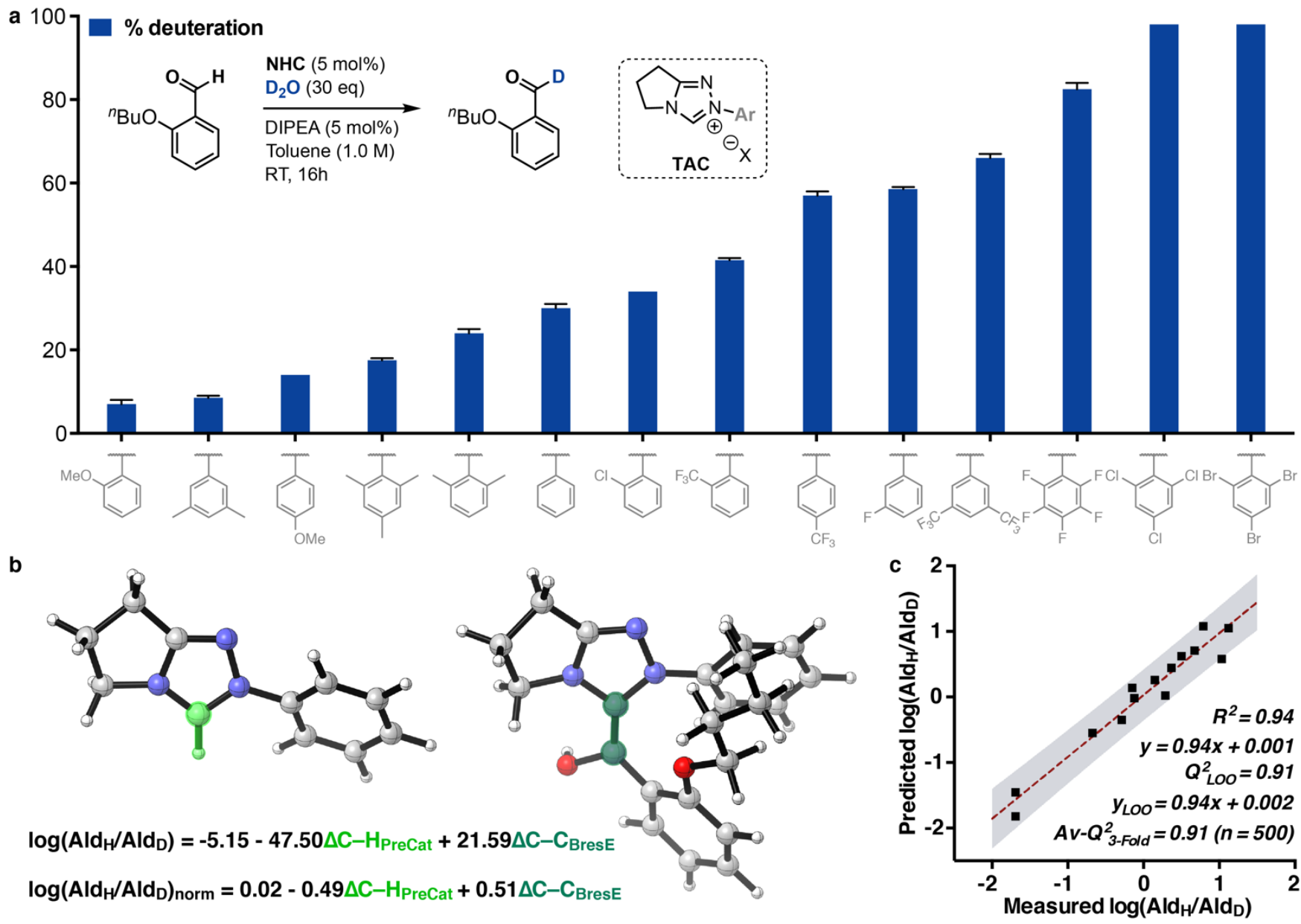

Figure 4. a. Percent of deuteration of $2^{-}{ }^{n}$ butoxy benzaldehyde with different triazolium catalysts. b. Equation for the deuteration ratio including two molecular descriptors: the difference in NBO charge between the carbene carbon and its proton in the precatalyst (left structure, light green), $\Delta \mathrm{C}-\mathrm{HPreCat}$, the difference in NBO charge between the carbene carbon and the aldehydic carbon after umpolung in the $\mathrm{E}$ isomer of the Breslow intermediate (right structure, dark green), $\Delta \mathrm{C}-\mathrm{C}_{\mathrm{BresE}}$ (normalized parameters were obtained by subtracting the mean and dividing by the standard deviation). c. Measured versus predicted log(ratio) of deuteration. Goodness-of-fit represented by $\mathrm{R}^{2}, \mathrm{Q}^{2}$ for a leave-one-out validation, and the average $\mathrm{Q}^{2}$ of a 3-fold validation performed 500 times on randomized sets, with $90 \%$ prediction interval in grey.

Accordingly, we added parameters derived from the relevant Breslow intermediates to our dataset. With this modification, we were able to identify a highly predictive model, which included only two parameters, one from the precatalyst structure and the other from the Breslow intermediate. The parameters were: (1) the precatalyst charge difference between the carbene carbon and its proton and (2) the charge difference between the same carbon and the adjacent one in the Breslow intermediate (figure 3.b.).

These parameters were respectively held to reflect the propensity of the pre-catalyst to enter the catalytic cycle by deprotonation and the nucleophilicity of the Breslow intermediate. Moreover, the different signs for each of the parameters in the equation were presumed to represent the requirement for a balance between a positively charged proton in the precatalyst structure and a negatively charged carbon in the Breslow intermediate to afford an effective catalyst. In both cases, it is the relative charge rather than the point charge that is best correlated to the outcome, which likely reflects the contribution of the catalyst backbone to these properties. These parameters cannot be considered strictly electronic because the torsion between the aryl and triazole rings highly influence their values. Interestingly, only parameters from the $\mathrm{E}$ isomer of the Breslow intermediate (figure 4.b. on the right) led to a highly predictive model, which indicates that the reaction pathway likely involves the $\mathrm{E}$ isomer (see computations SI for model with parameters derived from the $\mathrm{Z}$ isomer). This result highlights the aptitude of mathematical models to narrow down possible conformations that may participate in a reaction mechanism. ${ }^{44}$

With these results in hand, we were able to improve the deuterium incorporation of substrates that did not undergo full deuteration, namely, those with substituents at the 2position. These are known to form highly stable acyl anion adducts, thus more reactive catalysts were required for their full deuteration. On the other end of the substrate scope were substrates with highly reactive acyl anion adducts, namely, those with electron withdrawing groups at the $3^{-}$ and 4-position. These substrates were fully deuterated using SIPr likely due to their electrophilicity, the facilitated deprotonation of their acyl anion adducts, and their formation of highly electrophilic Breslow intermediates. 


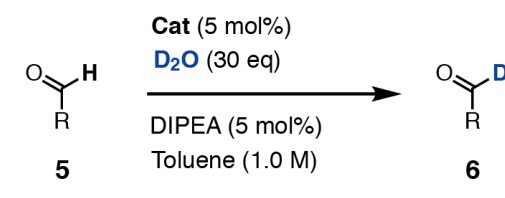

b

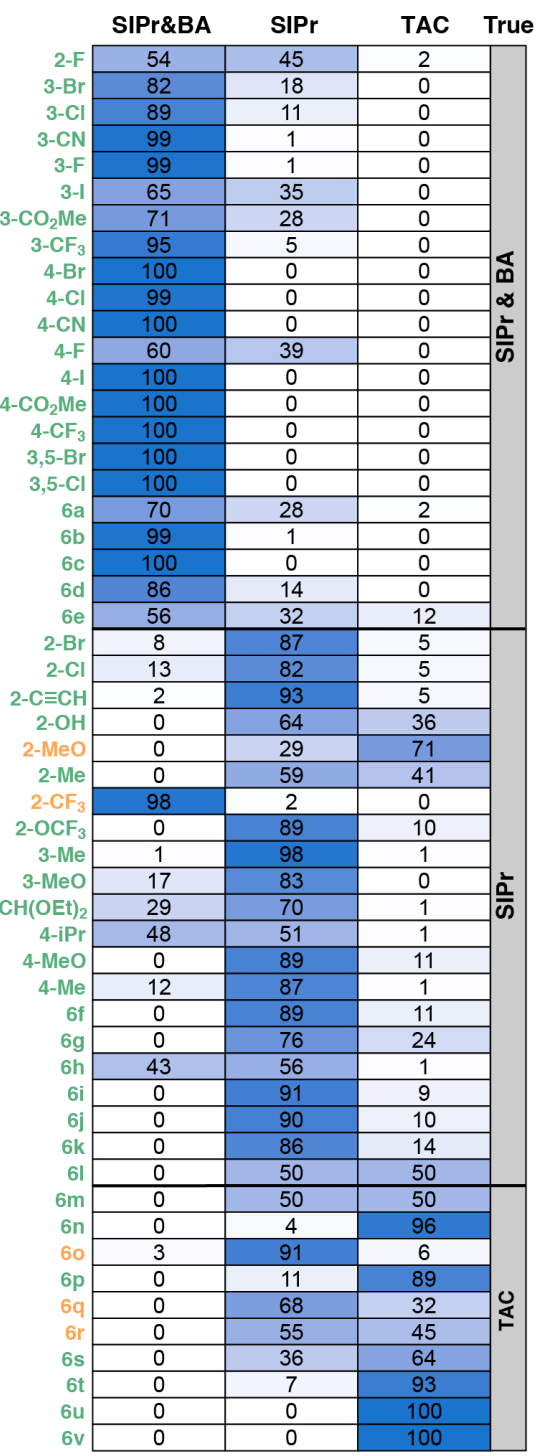

$\%$ probability

d

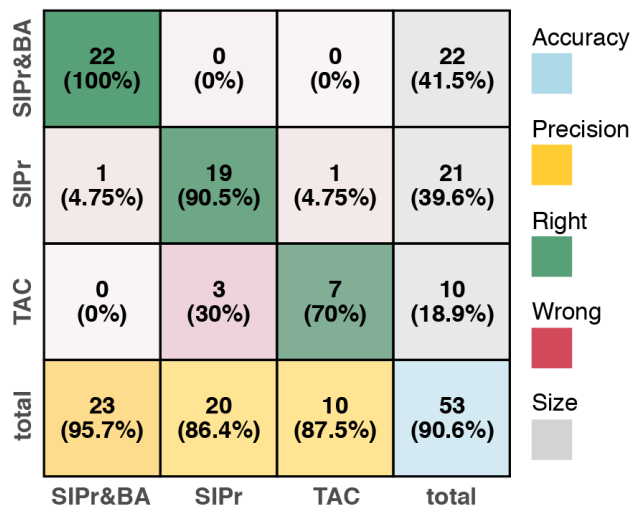

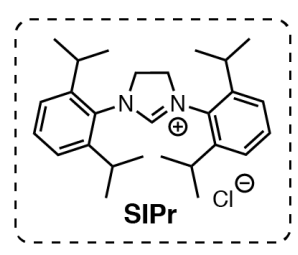

SIPr \& BA
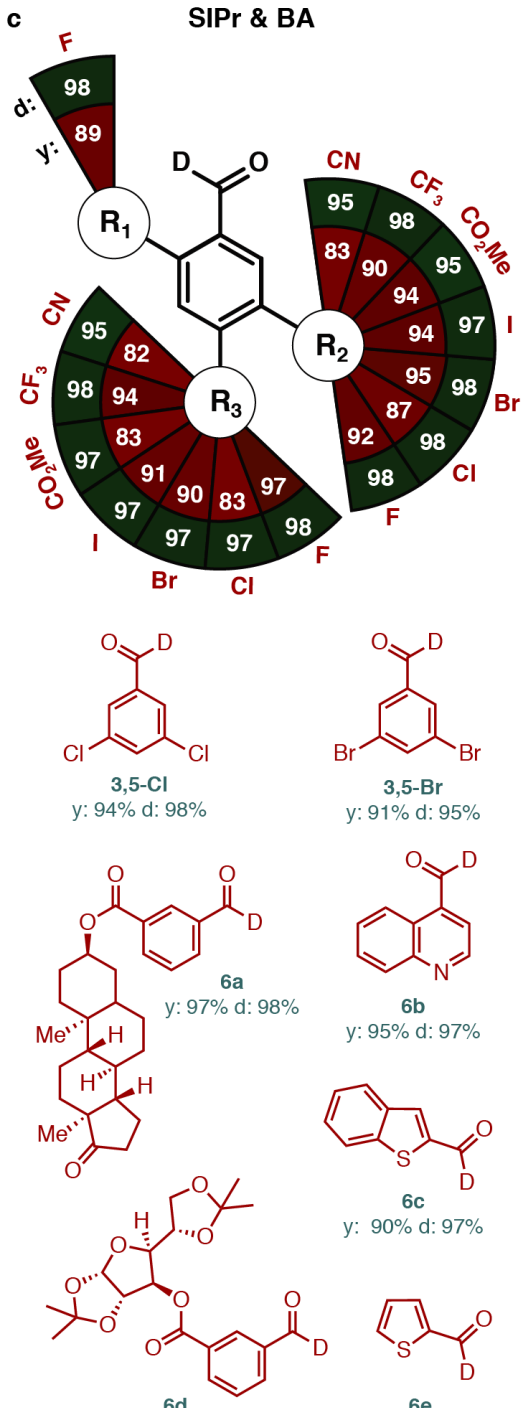

y: $91 \%$ d: $95 \%$

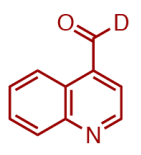

$6 \mathrm{~b}$

y: $95 \%$ d: $97 \%$

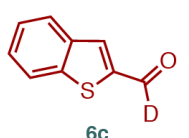

y: $90 \%$ d: $97 \%$

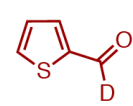

$$
6 \mathrm{e}
$$
y: $93 \%$ d: $98 \%$

\section{TAC $_{\mathrm{F}}$}
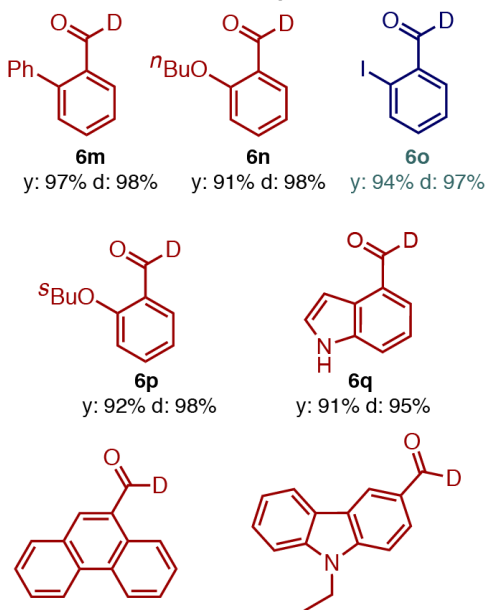

$6 r$

y: $86 \%$ d: $97 \%$

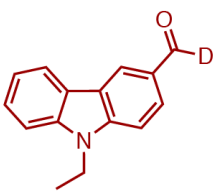

y: $97 \%$ d: $98 \%$

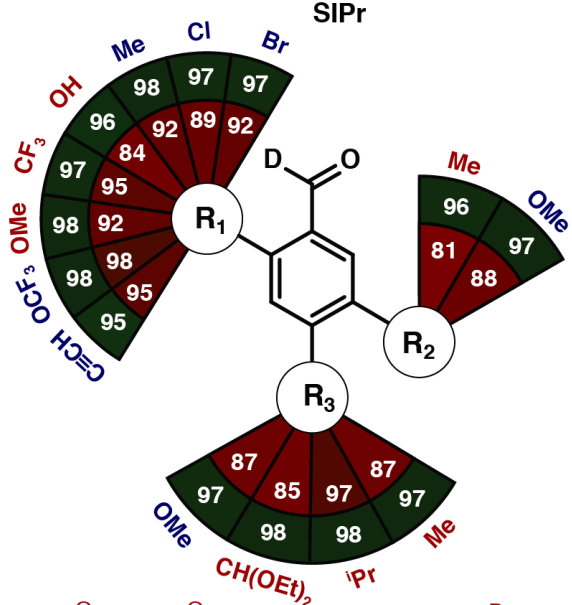

$\underbrace{6 f}_{y: 93 \% d: 98 \%}$

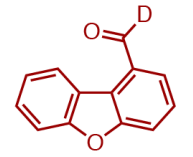

6

y: $98 \%$ d: $98 \%$<smiles>[2H]C(=O)c1cccc(C(=O)O[C@H]2CC(C)CC[C@H]2C(C)C)c1</smiles>

$\mathrm{C}_{C}^{C}$ : $98 \%$ d: $98 \%$ ee retained y: $98 \%$ d: $98 \%$<smiles>[2H]C(=O)c1cccc(OC(=O)[C@H](C)c2ccc3cc(OC)ccc3c2)c1</smiles>
y: $96 \%$ d: $98 \%$ y: $98 \%$ d: $99 \%$

Outliers

$\mathrm{TAC}_{\mathrm{Br}}$

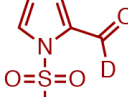<smiles>Cc1ccccc1</smiles>

6 t y: $92 \%$ d: $98 \%$<smiles>O=C(O)c1ccc[nH]1</smiles>
64 y: $74 \%$ d: $95 \%$

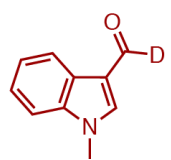
y: $91 \%$ d: $97 \%$

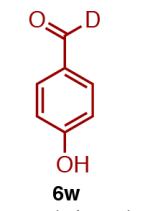

y: $97 \%$ d: $95 \%$

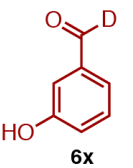

y: $91 \% d: 95 \%$

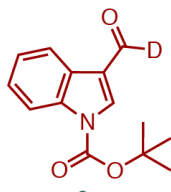
$90 \%$ d: $98 \%$ 
Figure 5. a. General reaction conditions for the deuteration of different aromatic aldehydes b. Probability table for the prediction of reaction conditions based on a multinomial logistic regression classification using four molecular descriptors: the aldehydic $\mathrm{C}=\mathrm{O}$ stretching frequency, the difference in NBO charge between the aldehydic carbon and oxygen, and the length and maximal width of the aldehyde (normalized parameters were obtained by subtracting the mean and dividing by the standard deviation and the prediction was cross-validated through a 3 -fold validation that was repeated over 500 iterations, see computations SI for details). The model produces the values in the columns, which represent the probability of each of the three possible reactions conditions to be optimal for a given substrate. Well predicted examples appear in green, while ill predicted ones appear in orange. c. Reaction scope divided according to the three categories of the classification (substrate scope plot modified from these references ${ }^{35,36}$ ). Reactions of substrates in red were performed at $50^{\circ} \mathrm{C}$ and those in blue were performed at $20^{\circ} \mathrm{C}$. Names and reaction outcomes in teal represent reactions performed in the presence of BA. y: yield, d: deuteration. d. Confusion matrix for the classification model: the right column sums the actual examples for each class (in parentheses their percentage of the total sample) and the bottom row sums the predicted examples for each class. The recall of prediction (the percentage out of each actual class that is well predicted) is given in parentheses in each cell, whereas the precision of prediction (the percentage of the correctly predicted results out of the total predicted results in each category) is summarized in parentheses in the bottom row. The overall accuracy is provided in the bottom right corner in parentheses (for further details see computations SI).

However, the same properties that cause their increased reactivity also lead to their facile transformation into homocoupling benzoin products. A fine balance between the reactivity and stability of the acyl anion adduct was required to mitigate the formation of the benzoin product while facilitating deuteration. Based on our previous work, boronic acids serve as secondary sphere modifiers to NHC catalysts, leading to stabilized reactive intermediates and slower, selective and more controlled benzoin reactions. Thus, we speculated that in the presence of $\mathrm{D}_{2} \mathrm{O}$, the addition of boronic acids may stabilize the acyl anion adducts to the extent that would quell the formation of benzoin products. Indeed, the formation of benzoin was fully suppressed by adding phenyl boronic acid to 4-trifluoromethylbenzaldehyde, which led to $75 \%$ benzoin formation with SIPr alone.

Scope and condition selection guideline. Overall, through the use of more electrophilic catalysts for substrates that form stable acyl anion adducts and the use of phenyl boronic acid to stabilize the acyl anion adducts of highly reactive substrates, a broad and diverse substrate scope was deuterated with excellent selectivity and reactivity (see Figure 5). Due to the multifaceted nature of the synthetic problem, several conditions were applied to different substrates to find the fine balance required for their deuteration and for the suppression of benzoin formation (Figure 5.a.). It should be noted, however, that minimal optimization was required due to our initial analysis of the requirements for each substrate class. Broadly, substrates with electron donating substituents at the 3- and 4-position could be fully deuterated with SIPr alone, while ones with electron withdrawing substituents required the addition of phenyl boronic acid to suppress the benzoin reaction. SIPr was also found to be efficient in the deuteration of many 2-substituted substrates; however, a more reactive catalyst was applied for 2substituted substrates that were not fully deuterated. In all cases the formation of the benzoin product was fully suppressed, yields were excellent and the level of deuteration was $>95 \%$ after isolation. In addition, we prepared and measured two enantioenriched examples that demonstrated no epimerization under our reaction conditions.

Previous methodologies for aldehyde deuteration using NHC catalysts suffer from several drawbacks that have been addressed in this work. Herein, catalytic amounts of base were used, while in previous work at least an equivalent of base was used. ${ }^{21-23}$ We fully suppressed the formation of the benzoin product even for electron-withdrawing substrates, while in previous work the benzoin product was often separated through column chromatography. Nevertheless, similarly to previous work, we did not provide one catalyst to address all substrates. For example, Chen, Zhang, Wang and coworkers published multiple catalysts and conditions (temperature, solvents, bases) for deuterating different substrates, which required an extensive trial-and-error optimization for each new example. ${ }^{22}$ As with many other organocatalytic $\mathrm{NHC}$ reactions, this requirement originated from the inherent characteristics of the different substrate-NHC intermediates. Furthermore, since the reactivity trend was not entirely steric or electronic, it has so far been difficult to formulate a clear guideline as to which conditions would work for new substrates. From the outset, we decided to limit the conditions and catalysts used with the aim of producing a comprehensive guideline for future deuterations of aromatic aldehydes. We only used three commonly employed catalyst structures and applied modest variations to temperature or base loading (see SI for further details). The conditions that were modified throughout this work are: temperature (20 or $50{ }^{\circ} \mathrm{C}$ ), catalyst (SIPr or $\mathrm{TAC}_{\mathrm{F} / \mathrm{Br}}$ ), and additive (using phenyl boronic acid or not).

In order to develop a more subtle guideline of the conditions required for each substrate, a logistic regression classification algorithm was applied to the substrate scope. We decided to classify the substrates into three broad categories: (1) those that undergo full deuteration with SIPr, but require boronic acid to suppress the formation of benzoin, (2) those that undergo full deuteration with SIPr alone, and (3) those that require a triazolium catalyst (TAC) for their full deuteration. These categories represent the reactivity of the substrates. The most reactive substrates easily undergo deuteration and benzoin reactions using SIPr, and therefore, require the addition of boronic acid to further stabilize the acyl anion and impede the benzoin reaction. The least reactive substrates that form the most stable acyl anion adducts require more reactive TAC catalysts for their full deuteration.

Using molecular features that describe the different substrates, classification models were tested with the aim of assigning substrates to each of these three categories and producing the probability of each category to provide the optimal conditions for a given substrate (see SI and Figure 5.b.). A predictive model was identified with the following four features: the aldehydic $\mathrm{C}=\mathrm{O}$ stretching frequency, the difference in NBO charge between the aldehydic oxygen and carbon, and the Sterimol maximal width and length of the substrate (see computations SI figure 10). The model showed 91\% accuracy and was cross-validated using a threefold validation (see computations SI for details). Notably, several of the substrates that were not well predicted, had in fact required slightly modified conditions, which could explain why the model did not classify them effectively. 

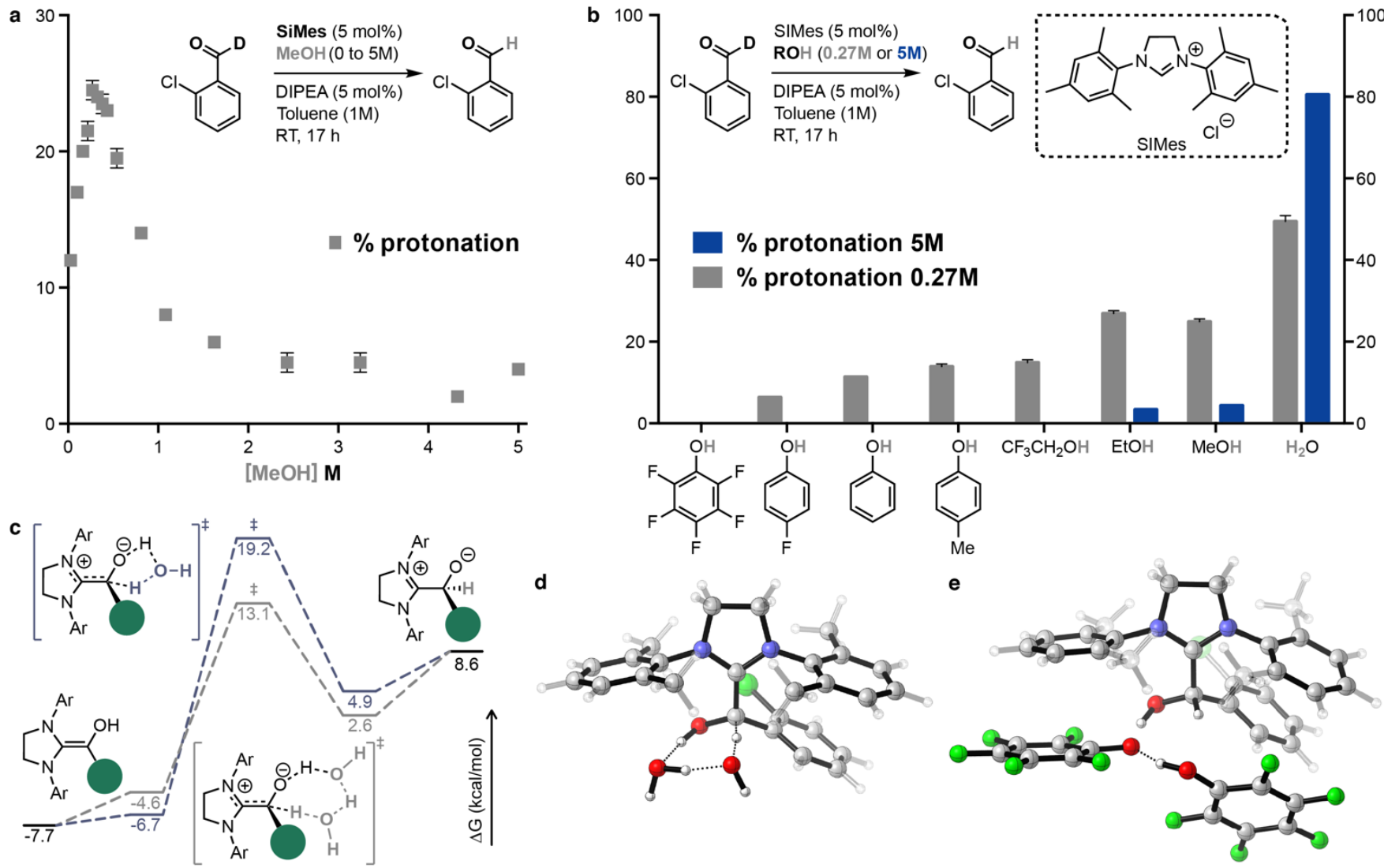

Figure 6. a. Protonation of deuterated 2-Cl-benzaldehyde using different concentrations of methanol as protonating reagent. b. Protonation of deuterated 2-Cl-benzaldehyde with different protonating reagents at concentrations of ${ }_{5} \mathrm{M}$ and $0.27 \mathrm{M}$ ( $\mathrm{x}$ axis arranged by increasing pK $\mathrm{K}_{\mathrm{a}}$ values). c. Gibbs free energy profile of the deuteration step through a Grotthus-type mechanism with one (blue) or two bridging water molecules (grey). The structures of the intermediates are included in the SI. For simplicity, the computations were performed using a 2,6-methyl substituted aryl group. d. Transition-state structure of the proton transfer step with two bridging water molecules. e. Structure of the pentafluo-ro phenolate stabilized acyl anion intermediate.

For example, 2-MeO- and 2-CF3-benzaldehyde are not well predicted with SIPr alone, and in both cases the amount of base was doubled (10 $\mathrm{mol} \%$ instead of $5 \mathrm{~mol} \%$ ) to afford fully deuterated aldehydes. Furthermore, 2-I-benzaldehyde, which was predicted to require $\mathrm{SIPr}$, actually required TAC combined with BA, indicating that TAC is slightly over reactive and forms benzoin, while SIPr is slightly under reactive and does not lead to full deuteration.

Overall, this model provides a detailed guideline for future deuteration reactions using easily extracted features that represent the steric and nucleophilic characteristics of the aldehydes. Likewise, the probabilities given for each condition (Figure 5.b.) provide a reliability gauge of the prediction for each substrate. Three examples were excluded from this analysis as outliers: 4-hydroxybenzaldehyde (6w) that required a different solvent due to solubility issues in toluene; 3-hydroxybenzaldehyde (6x) that required a protecting group that was removed under reaction conditions also due to solubility issues; and ${ }^{t}$ butoxycarbonyl protected 3 -indole (6y) that required 1.5 equivalents of boronic acid to fully suppress the competing benzoin reaction. These challenging examples provide an additional guideline for substrates that may not be fully deuterated under other conditions, but can still be addressed applying the herein proposed precepts.

Using the above-mentioned conditions, several new deuterated compounds were prepared and improved yields and deuteration levels were achieved across the board, most no- tably for electron-withdrawing or sterically hindered benzaldehydes. In all cases, the formation of the benzoin product was fully suppressed, providing a highly selective reaction manifold. The developed conditions are also suitable for latestage deuteration as the yield and selectivity are unprecedented and the amount of base used is catalytic. To further increase its practicality, the reaction was performed on a large scale. We used 2-Cl-benzaldehyde as a model substrate on an $11 \mathrm{mmol}$ scale and attained $97 \%$ deuterium incorporation and $88 \%$ isolated yield with $5 \mathrm{~mol} \%$ base and $5 \mathrm{~mol} \%$ SIPr catalyst at room temperature. Following a short optimization (see experimental SI table 25), we were able to perform the reaction with extremely low loadings of $0.1 \mathrm{~mol} \%$ base and $0.1 \mathrm{~mol} \% \mathrm{TAC}_{\mathrm{Br}}$ catalyst at $90{ }^{\circ} \mathrm{C}$, affording $98 \%$ deuterium incorporation and $96 \%$ isolated yield.

With the conditions-selecting guideline in hand, we wished to further understand the mechanism of the reaction and gain insight on the cause different aldehyde substitution patterns necessitate modified reaction conditions. We hypothesized that the deuteration occurred either as a nucleophilic attack of the Breslow intermediate on a water molecule, as previously suggested, ${ }^{22-23}$ or through a Grotthus-type mechanism, by which protons diffuse through a hydrogen bond network. ${ }^{45}$ The latter would entail the concomitant formation and cleavage of covalent bonds between the Breslow intermediate and one or more water molecules. For practical reasons, we studied this effect experimentally by proto- 
nating a deuterated aldehyde using different alcohols as proton sources. It was assumed that if it were a nucleophilic attack, alcohols with lower pKa values would lead to improved protonation efficiencies. However, we found that under our reaction conditions with $5 \mathrm{M}$ of the proton source, all the alcohols tested led to only trace amounts of protonation (Figure 6.b. blue bars). We presumed this result indicated that the reaction proceeded through a Grotthus-type mechanism, which we anticipated to be less effective for alcohols compared to water due to steric hindrance. To support this claim, the complete reaction mechanism was studied by density functional theory (DFT) computations. The results indicated that a Grotthus-type mechanism using two bridging water molecules was the lowest energy path (Figure 6c-e, see computations SI for the full mechanism).

However, once the same computation was performed with bridging methanol molecules, a similar energy profile was identified, both in terms of molecular structures and energies. Based on this computational result the protonation level should be similar using methanol or water, yet the experimental result revealed only traces of protonation using methanol as protonating agent. We hypothesized that the solubility of methanol and immiscibility of water in toluene could be the possible source of this theory-experiment contradiction. Thus, different concentrations of methanol were tested experimentally to probe whether the reaction would work by matching the effective concentration of water in toluene, which is estimated between $0.2-0.5 \mathrm{M}$ at room temperature. ${ }^{46}$ Indeed, the protonation of deuterated aldehyde reached a maximum at this concentration range (Figure 6a) supporting the validity of the computed Grotthus-type mechanism for methanol. The diminished activity at higher methanol concentrations is attributed to alcohols' micellelike aggregation processes in non-polar solvents, which hampers methanol's availability. ${ }^{47-48}$ This finding encouraged us to run the protonation reaction with all the previously tested alcohols at the concentration that led to the maximal protonation with methanol (grey bars in Figure 6b). Though improved reactivity was observed compared to the $5 \mathrm{M}$ conditions, alcohols with decreased $\mathrm{pK}_{\mathrm{a}}$ values, which were presumed to more readily transfer their proton to the Breslow intermediate, still afforded decreased protonation values. It was unclear whether perhaps steric factors could lead to these lower deuteration rates as several of the alcohols with lower $\mathrm{pK}_{\mathrm{a}}$ values were also bulkier. Computations revealed that this was not the case and, in fact, up to a certain $\mathrm{pK}_{\mathrm{a}}$ the mechanism with two bridging molecules is the lowest energy path; but from ethanol onwards, the lower $\mathrm{pK}_{\mathrm{a}}$ values enable an increasingly strong stabilization of the acyl anion adduct by an alcoholate, creating a thermodynamic sink that diminishes the efficiency of deuteration (Figure 6e and SI).

In summary, we demonstrated the utility of controlling the stability of reactive intermediates in NHC organocatalysis. The stabilization of the acyl anion adducts provided an entry point for the facile deuteration of aldehydes in the presence of $\mathrm{D}_{2} \mathrm{O}$, leading to unprecedented deuterium incorporation while fully suppressing the formation of the benzoin product. The mechanistic basis for this reaction design process led not only to improved reaction conditions and lower catalyst and base loading, but more importantly, it aided in the selection of appropriate conditions for different aldehydes. The identification of a limited set of conditions for different substrate classes facilitated the construction of a machine-learning based guideline for selecting the catalyst and conditions required for deuterating substrates with diverse substitution patterns.

\section{ASSOCIATED CONTENT}

\section{Supporting Information}

The Supporting Information is available free of charge.

\section{AUTHOR INFORMATION}

\section{Corresponding Author}

Correspondence can be addressed to anatmilo@bgu.ac.il.

\section{Author Contributions}

${ }^{\ddagger}$ These authors contributed equally.

\section{ACKNOWLEDGMENT}

This research was supported by the Israel Science Foundation (Grant No. 1193/17). We thanks Doron Pappo for fruitful discussions. V.D. gratefully acknowledges the PBC for a postdoctoral fellowship. S.C.G. gratefully acknowledges the Kreitman Graduate School for a postdoctoral fellowship.

\section{REFERENCES}

(1) Holland, M. C.; Gilmour, R., Deconstructing covalent organocatalysis. Angew. Chem. Int. Ed. 2015, 54 (13), 3862-71.

(2) List, B., Introduction: Organocatalysis. Chem. Rev. 2007, 107 (12), 5413-5415

(3) Jacobsen, E. N.; MacMillan, D. W., Organocatalysis. Proc. Natl. Acad. Sci. 2010, 107 (48), 20618-9.

(4) Dalko, P. I.; Moisan, L., In the golden age of organocatalysis. Angew. Chem. Int. Ed. Engl. 2004, 43 (39), 5138-75.

(5) MacMillan, D. W., The advent and development of organocatalysis. Nature 2008, 455 (7211), 304-8.

(6) Gaunt, M. J.; Johansson, C. C. C.; McNally, A.; Vo, N. T., Enantioselective organocatalysis. In Drug Discov. Today, 2007; Vol. 12, pp 8-27.

(7) Barbas, C. F., Organocatalysis Lost: Modern Chemistry, Ancient Chemistry, and an Unseen Biosynthetic Apparatus. Angew. Chem. Int. Ed. 20o8, 47 (1), 42-47.

(8) Katsnelson, A., Heavy drugs draw heavy interest from pharma backers. Nat. Med. 2013, 19 (6), 656.

(9) Gant, T. G., Using deuterium in drug discovery: leaving the label in the drug. J. Med. Chem. 2014, 57 (9), 3595-611.

(10) Atzrodt, J.; Derdau, V.; Kerr, W. J.; Reid, M., Deuterium- and Tritium-Labelled Compounds: Applications in the Life Sciences. Angew. Chem. Int. Ed. 2018, 57 (7), 1758-1784.

(11) Russak, E. M.; Bednarczyk, E. M., Impact of Deuterium Substitution on the Pharmacokinetics of Pharmaceuticals. Ann. Pharmacother. 2018, 53 (2), 211-216.

(12) Scheppele, S. E., Kinetic isotope effects as a valid measure of structure-reactivity relations. Isotope effects and nonclassical theory. Chem. Rev. 1972, 72 (5), 511-532.

(13) Busenlehner, L. S.; Armstrong, R. N., Insights into enzyme structure and dynamics elucidated by amide H/D exchange mass spectrometry. Arch. Biochem. Biophys. 2005, 433 (1), 34-46.

(14) Atzrodt, J.; Derdau, V.; Fey, T.; Zimmermann, J., The renaissance of H/D exchange. Angew. Chem. Int. Ed. 2007, 46 (41), 7744-65. 
(15) Isbrandt, E.; Vandavasi, J.; Zhang, W.; Jamshidi, M.; Newman, S., Catalytic Deuteration of Aldehydes with $\mathrm{D}_{2} \mathrm{O}$. Synlett 2017, 28 (20), 2851-2854.

(16) Kerr, W. J.; Reid, M.; Tuttle, T., Iridium-Catalyzed FormylSelective Deuteration of Aldehydes. Angew. Chem. Int. Ed. 2017, 129 (27), 7916-7920.

(17) Ibrahim, M. Y. S.; Denmark, S. E., Palladium/Rhodium Cooperative Catalysis for the Production of Aryl Aldehydes and Their Deuterated Analogues Using the Water-Gas Shift Reaction. Angew. Chem. Int. Ed. 2018, 57 (32), 10362-10367.

(18) Li, X.; Wu, S.; Chen, S.; Lai, Z.; Luo, H. B.; Sheng, C., OnePot Synthesis of Deuterated Aldehydes from Arylmethyl Halides. Org. Lett. 2018, 20 (7), 1712-1715.

(19) Zhang, M.; Yuan, X.-A.; Zhu, C.; Xie, J., Deoxygenative Deuteration of Carboxylic Acids with $\mathrm{D}_{2} \mathrm{O}$. Angew. Chem. Int. Ed. 2018, 58 (1), 312-316.

(20) Dong, J.; Wang, X.; Wang, Z.; Song, H.; Liu, Y.; Wang, Q., Formyl-selective deuteration of aldehydes with $\mathrm{D} \quad 2 \mathrm{O}$ viasynergistic organic and photoredox catalysis. Chem. Sci. 2020, 11 (4), 1026-1031.

(21) Liu, W.; Zhao, L.-L.; Melaimi, M.; Cao, L.; Xu, X.; Bouffard, J.; Bertrand, G.; Yan, X., Mesoionic Carbene (MIC)-Catalyzed H/D Exchange at Formyl Groups. Chem 2019, 5 (9), 2484-2494.

(22) Geng, H.; Chen, X.; Gui, J.; Zhang, Y.; Shen, Z.; Qian, P.; Chen, J.; Zhang, S.; Wang, W., Practical synthesis of $C_{1}$ deuterated aldehydes enabled by NHC catalysis. Nat. Catal. 2019, 62, 1-7.

(23) Sawama, Y.; Miki, Y.; Sajiki, H., N-Heterocyclic Carbene Catalyzed Deuteration of Aldehydes in $\mathrm{D}_{2} \mathrm{O}$. Synlett 2020, 31 (07), 699-702.

(24) Santanilla, A. B.; Regalado, E. L.; Pereira, T.; Shevlin, M.; Bateman, K.; Campeau, L. C.; Schneeweis, J.; Berritt, S.; Shi, Z. C.; Nantermet, P.; Liu, Y.; Helmy, R.; Welch, C. J.; Vachal, P.; Davies, I. W.; Cernak, T.; Dreher, S. D., Nanomole-Scale HighThroughput Chemistry for the Synthesis of Complex Molecules. Science 2015, 347 (6217), 49-53.

(25) Dhayalan, V.; Gadekar, S. C.; Alassad, Z.; Milo, A., Unravelling mechanistic features of organocatalysis with in situ modifications at the secondary sphere. Nat. Chem. 2019, 11 (6), 543-551.

(26) Breslow, R., Rapid Deuterium Exchange in Thiazolium Salts1. J. Am. Chem. Soc. 1957, 79 (7), 1762-1763.

(27) Breslow, R., On the Mechanism of Thiamine Action. IV.1Evidence from Studies on Model Systems. J. Am. Chem. Soc. 1958, 8 o (14), 3719-3726.

(28) Kluger, R.; Tittmann, K., Thiamin diphosphate catalysis: enzymic and nonenzymic covalent intermediates. Chem. Rev. 2008, 108 (6), 1797-833.

(29) Enders, D.; Niemeier, O.; Henseler, A., Organocatalysis by N-Heterocyclic Carbenes. Chem. Rev. 2007, 107 (12), 5606-5655.

(30) Enders, D.; Henseler, A., A Direct Intermolecular CrossBenzoin Type Reaction: N-Heterocyclic Carbene-Catalyzed Coupling of Aromatic Aldehydes with Trifluoromethyl Ketones. Adv. Synth. Catal. 2009, 351 (11-12), 1749-1752.

(31) Enders, D., N-heterocyclic carbene catalysed asymmetric cross-benzoin reactions of heteroaromatic aldehydes with trifluoromethyl ketones. Chem. Commun. 2010, 46 (34), 6282.

(32) Breslow, R., On the Mechanism of Thiamine Action. IV. 1 Evidence from Studies on Model Systems. J. Am. Chem. Soc. 1958, 8 o (14), 3719-3726.

(33) Berkessel, A.; Elfert, S.; Yatham, V. R.; Neudörfl, J.-M.; Schlörer, N. E.; Teles, J. H., Umpolung by N-Heterocyclic Carbenes: Generation and Reactivity of the Elusive 2,2-Diamino Enols (Breslow Intermediates). Angew. Chem. Int. Ed. 2012, 51 (49), 12370-12374.

(34) Collett, C. J.; Massey, R. S.; Maguire, O. R.; Batsanov, A. S.; O'Donoghue, A. C.; Smith, A. D., Mechanistic Insights into the
Triazolylidene-Catalysed Stetter and Benzoin Reactions: Role of the N-Aryl Substituent. Chem. Sci. 2013, 4 (4), 1514-1522.

(35) Collett, C. J.; Massey, R. S.; Taylor, J. E.; Maguire, O. R.; O'Donoghue, A. C.; Smith, A. D., Rate and Equilibrium Constants for the Addition of $\mathrm{N}$-Heterocyclic Carbenes into Benzaldehydes: A Remarkable 2-Substituent Effect. Angew. Chem. Int. Ed. 2015, 127 (23), 6991-6996.

(36) Verloop, A.; Hoogenstraaten, W.; Tipker, J., Development and Application of New Steric Substituent Parameters in Drug Design. In Drug design: Volume VII, Academic Press, Inc.: 1976; pp 165-207.

(37) Berkessel, A.; Elfert, S.; Yatham, V. R.; Neudörfl, J.-M.; Schlörer, N. E.; Teles, J. H., Umpolung by N-Heterocyclic Carbenes: Generation and Reactivity of the Elusive 2,2-Diamino Enols (Breslow Intermediates). In Angew. Chem. Int. Ed., 2012; Vol. 51, pp 12370-12374.

(38) Flanigan, D. M.; Romanov-Michailidis, F.; White, N. A.; Rovis, T., Organocatalytic Reactions Enabled by N-Heterocyclic Carbenes. Chem. Rev. 2015, 9307-9387.

(39) Chen, X.; Wang, H.; Jin, Z.; Chi, Y. R., NHeterocyclicCarbene Organocatalysis: Activation Modes and Typical Reactive Intermediates. Chin. J. Chem. 2020, 510, 485-36. (40) Mahatthananchai, J.; Bode, J. W., The Effect of the NMesityl Group in NHC-Catalyzed Reactions. Chem. Sci. 2012, 3 (1), 192-197.

(41) Mahatthananchai, J.; Bode, J. W., On the Mechanism of NHeterocyclic Carbene-Catalyzed Reactions Involving Acyl Azoliums. Acc. Chem. Res. 2014, 47 (2), 696-707.

(42) Higgins, E. M.; Sherwood, J. A.; Lindsay, A. G.; Armstrong, J.; Massey, R. S.; Alder, R. W.; O'Donoghue, A. C., pKas of the conjugate acids of $\mathrm{N}$-heterocyclic carbenes in water. Chem. Commun. 2011, 47 (5), 1559-1561.

(43) Massey, R. S.; Collett, C. J.; Lindsay, A. G.; Smith, A. D.; O'Donoghue, A. C., Proton Transfer Reactions of Triazol-3ylidenes: Kinetic Acidities and Carbon Acid pKa Values for Twenty Triazolium Salts in Aqueous Solution. J. Am. Chem. Soc. 2012, 134 (50), 20421-20432.

(44) Reid, J. P.; Sigman, M. S., Holistic prediction of enantioselectivity in asymmetric catalysis. Nature 2019, 571 (7765), 343-348.

(45) Neel, A. J.; Milo, A.; Sigman, M. S.; Toste, F. D., Enantiodivergent Fluorination of Allylic Alcohols: Data Set Design Reveals Structural Interplay between Achiral Directing Group and Chiral Anion. J. Am. Chem. Soc. 2016, 138 (11), 38633875 .

(46) Santiago, C. B.; Milo, A.; Sigman, M. S., Developing a Modern Approach To Account for Steric Effects in HammettType Correlations. J. Am. Chem. Soc. 2016, 138 (40), 13424-13430. (47) Agmon, N., The Grotthuss mechanism. Chem. Phys. Lett. 1995, 244 (5), 456-462.

(48) https://srdata.nist.gov/solubility/IUPAC/SDS-37/SDS-37pages 369.pdf

(49) Essafri, I.; Ghoufi, A., Microstructure of nonideal methanol binary liquid mixtures. Phys. Rev. E 2019, 99 (6-1), 062607.

(50) Mhanna, R.; Lefort, R.; Noirez, L.; Morineau, D., Microstructure and concentration fluctuations in alcoholToluene and alcohol-Cyclohexane binary liquids: A small angle neutron scattering study. J. Mol. Liq. 2016, 218, 198-207. 